\title{
Modeling a Mechanical Molecular Spring Isolator with High-Static-Low-Dynamic-Stiffness Properties
}

\author{
Zhanyong Li, ${ }^{1}$ Qian Chen $\mathbb{D}^{1},{ }^{1}$ Fengshou Gu, ${ }^{2}$ and Andrew Ball ${ }^{2}$ \\ ${ }^{1}$ State Key Laboratory of Mechanics and Control of Mechanical Structures, Nanjing University of Aeronautics and Astronautics, \\ Nanjing 210016, China \\ ${ }^{2}$ The Centre for Efficiency and Performance Engineering, University of Huddersfield, Huddersfield, UK \\ Correspondence should be addressed to Qian Chen; q.chen@nuaa.edu.cn
}

Received 30 March 2020; Revised 1 June 2020; Accepted 8 July 2020; Published 3 August 2020

Academic Editor: Stefano Marchesiello

Copyright (c) 2020 Zhanyong Li et al. This is an open access article distributed under the Creative Commons Attribution License, which permits unrestricted use, distribution, and reproduction in any medium, provided the original work is properly cited.

\begin{abstract}
A mechanical molecular spring isolator (MMSI) is proposed for the purpose of isolating the low-frequency vibration of a heavy payload. The MMSI is a passive vibration isolation technique mimicking molecular spring isolator characteristics of high-staticlow-dynamic stiffness (HSLDS). An MMSI consists of a piston-cylinder container filled with the liquid and some hydraulic spring accumulators. The piston would support a lump of mass and be subjected to a specific external vibration excitation force. Those accumulators can get intercommunication by the liquid to produce the transformation from high static stiffness to low dynamic stiffness. The stiffness model of the MMSI with several identical accumulators is established based on the hydrostatic law. After that, some parameters that significantly influence the stiffness characteristics are studied. Results show that the stiffness property of this kind of MMSI demonstrates a piecewise linearity of three segments. It applies the averaging method to acquire amplitudefrequency and phase-frequency relationships of the piecewise linear vibration isolation system. An inevitable jump phenomenon may occur when the exciting force reaches the critical value. The vibration isolation performance is evaluated by energy transmissibility. Finally, an experimental prototype was designed to carry out quasi-static and dynamic experiments to verify the stiffness model and the dynamic properties as an HSLDS vibration isolator.
\end{abstract}

\section{Introduction}

Vibration isolation has been increasingly demanded for high-speed vehicles, precision machinery, and megastructures as a result of the higher fatigue endurance for structures and higher human comfort expectations in both static and dynamic environments. A variety of vibration protection means are continuously improving to accommodate these new standards. Passive vibration control technology would always capture engineers' enthusiasm for its low costs and reliable vibration isolation performance [1-3]. Concerning an ideal case where a lump of mass $m$ is supported by a linear spring with stiffness $k$ on a rigid foundation, effective vibration isolation of this linear system can be achieved when the excitation frequency $\omega$ is greater than $\sqrt{2 \mathrm{k} / \mathrm{m}}$. A lower stiffness of the supporting linear spring is required for isolating a lower frequency vibration. As a result, the linear spring will inevitably produce a higher static deformation, which may bring about more vertical installation space of the system and worse lateral stability of the linear spring. It causes the dilemma that low natural frequency and small static deformation cannot be achieved at the same time for a traditional linear isolator. Particularly, the dilemma is more remarkable on the heavy payload lowfrequency vibration isolation field.

The nonlinear passive vibration isolators with the HSLDS property can easily break through this dilemma [4]. This kind of isolators only requires a smaller deformation while offering a lower stiffness when the payload is at the equilibrium position compared to the traditional isolator. In the recent decade, the study of this type of passive vibration isolators has been progressed. On the one hand, the most prevalent solution is to develop a negative stiffness device to combine with a positive stiffness element (such as the 
traditional linear spring). Due to the diversity of the negative stiffness device mechanisms, there exist plentiful nonlinear passive vibration isolators with the HSLDS property. Brennan's research group [5-8] developed two different types of nonlinear isolators. The first one consists of a vertical linear spring and two oblique springs; the second one is configured with coil springs and attracting magnets. Huang et al. [9] applied Euler-bulked beams as the negative stiffness element. Zhou et al. [10] utilized cam-roller-spring mechanisms as the negative stiffness element. Sun and Jing [11] found that scissor-like structures may also bring in the negative stiffness. Besides, Mofidian and Bardaweel [12] employed the magnetic spring as the positive stiffness element connecting with oblique springs to form a different isolator with the HSLDS property. They all are able to achieve a quasi-zero stiffness vibration isolator by properly choosing system parameters. The aforementioned nonlinear isolators have been proved to be superior on isolation performance than traditional linear springs. However, on account of those mechanisms' geometrical and physical nonlinearities, some harmful nonlinear dynamic phenomena (the most common one is the sudden jump) would inevitably occur around the equilibrium position, and they get more severe as the magnitude of the excitation force increases. Besides, because of the connection of positive stiffness and negative stiffness elements, those isolators are normally with big size and complicated structures.

On the other hand, Chen's research group developed one type of solid and liquid mixture (SALiM) vibration isolators for isolating the low-frequency vibration of massive machinery. The SALiM consists of the almost incompressible liquid and a number of compressible elastic solid elements. When under shock or vibration, the incompressible liquid can instantly pass the pressure on to all elastic solid elements, which causes all elastic solid elements to be compressed and deformed simultaneously. As a result, it could greatly absorb and dissipate the energy of vibrations or shock. The SALiM vibration isolator possesses a high loading capacity and a high stability of performance; it is especially aimed for the heavy payload low-frequency vibration isolation purpose. Previous work mainly focuses on the solid elastic element and so does the research of this article Teng and Chen [13] applied hollow rubber spheres as the elastic element and estimated the vibration isolation performance of the SALiM isolator. Gao et al. [14] took advantage of the U-shaped bellows as the elastic element to develop the B-SALiM isolator, and they denoted that the isolator can be modified as a nonlinear isolator with the HSLDS property. Inspired by the aforementioned work, $\mathrm{Yu}$ et al. $[15,16]$ found that a kind of nanoporous hydrophobic nanofunctional materials, which can absorb and release some water until the hydraulic pressure reaches a certain value, can be applied to the solid elastic element. Therefore, the molecular spring isolator based on nanoporous hydrophobic nanofunctional materials is developed, and it naturally presents the HSLDS property. Moreover, its stiffness exhibits nearly a long linearity around the static equilibrium position. Because of the characteristics of high density and high water absorption of the nanoporous hydrophobic nanofunctional materials, the molecular spring isolator owns a very small structure size.

Inspired by the distinct mechanism for the HSLDS property of the molecular spring isolator, we noted that the nanoporous hydrophobic nanofunctional materials only absorb and release water until the hydraulic pressure reaches a certain value. It seems that naturally there exists a switch in this kind of material related to the hydraulic pressure. A conventional hydraulic spring accumulator may acquire that function artificially. So we substitute the particular material of the molecular spring isolator with some hydraulic spring accumulators to form the mechanical molecular spring isolator (MMSI). Each hydraulic spring accumulator consists of a piston, a container, and a linear spring. The piston is sealed and is subjected to a force offered by the precompressed linear spring. As a result, the liquid cannot intrude into the accumulator until the hydraulic pressure reaches a certain value. The MMSI also possesses the HSLDS property. Besides, on account of the fact that the configuration of the accumulator is artificial, there is no necessity to utilize the specific water to get all accumulators intercommunicated. Any liquid is available; and the critical hydraulic pressure is adjustable, so the MMSI may not have to work at that high hydraulic pressure.

It is noticed that the configurations of all accumulators in the MMSI can be designed to be quite different from each other, which may result in a much more complicated stiffness property. In order to understand some simple and essential features of the MMSI, we assume that all accumulators are of the same configuration in this paper. The MMSI stiffness model is studied with the hydrostatic law based on that assumption, and it presents a piecewise linearity of three segments. The equation of motion of the MMSI isolation system is established at the center of the second segment. The averaging method is applied to investigate the primary resonance response of this kind of nonlinear vibration isolation system. The energy transmissibility is applied to evaluate the vibration isolation performance. An experimental prototype has been designed to carry out quasi-static and dynamic experiments to estimate the static and dynamic properties of the MMSI isolation system.

\section{MMSI Mechanical Model}

2.1. MMSI Stiffness Model. In analogy with the function of the molecular spring, we mimic the molecular spring with hydraulic spring accumulators and the liquid. The combination of hydraulic spring accumulators and the liquid is referred to as mechanical molecular spring (MMS) correspondingly. The MMS is sealed in the piston-cylinder container. The schematic diagram of an MMSI is presented in Figure 1(a). Define the MMSI piston diameter as $D$, the initial volume of the liquid as $V_{0}$, and the initial hydraulic pressure as $P_{0}$. Define $n$ as the number of those accumulators, and there is at least one accumulator; that is, $n \geq 1$. The structure of the accumulator is shown in Figure 1(b). The accumulator piston diameter is $d$, and it is 


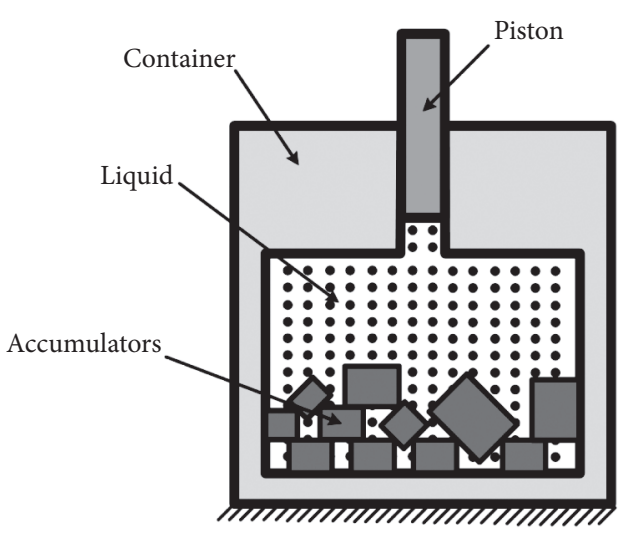

(a)

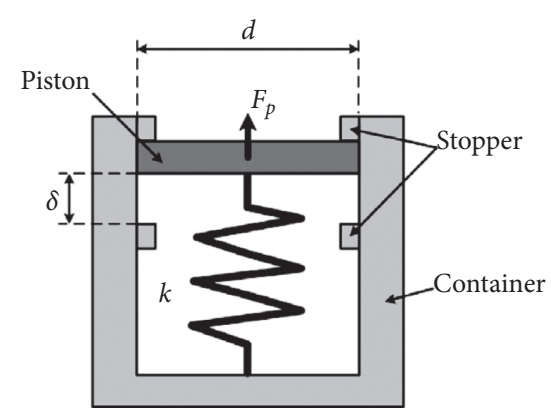

(b)

FIgURE 1: Experimental apparatus. (a) Schematic diagram of MMSI. (b) Hydraulic spring accumulator.

sealed and matched with the container. Meanwhile, the piston is subjected to a force offered by the precompressed linear spring whose stiffness is $k$, and the precompressed force is $F_{\mathrm{p}}$. The movement of the piston is confined by two stoppers, which results in a maximum displacement $\delta$ that the piston can reach. Hence, there exist two critical hydraulic pressures of the accumulator. The first critical hydraulic pressure is formed by the precompressed force, and the definition of the first critical hydraulic pressure is

$$
P_{c 1}=\frac{F_{\mathrm{p}}}{\pi \cdot d^{2} / 4}+P_{0}
$$

Meanwhile the second critical hydraulic pressure is produced by the finite displacement of the accumulator piston, so the second critical hydraulic pressure is defined as

$$
P_{c 2}=\frac{F_{\mathrm{p}}+k \cdot \delta}{\pi \cdot d^{2} / 4}+P_{0}
$$

Applying an external force $F$ onto the MMSI container piston would cause the MMSI piston to have a vertical displacement $x$. Then the hydraulic pressure would become $P$, and the volume of the liquid would become $V$ correspondingly. Among them, the external force is related to the hydraulic pressure as

$$
F=\frac{\left(P-P_{0}\right) \cdot \pi \cdot D^{2}}{4} .
$$

According to the hydrostatic law, the volume compressibility of a particular liquid is a constant in a specific environment. Its magnitude is decided by the hydraulic pressure and the liquid volume as follows:

$$
\beta=-\frac{V-V_{0}}{V_{0}} \cdot \frac{1}{P-P_{0}} .
$$

Since this paper focuses on studying the MMSI with all identical accumulators, there are only two critical hydraulic pressures. Therefore, the hydraulic pressure starting from zero to infinity is divided into three segments. We define the segment $P<P_{c 1}$ as the bearing segment. There the hydraulic pressure is not able to overcome the precompressed force. So the displacement of the MMSI piston is only produced by the volume change of the liquid. Then the vertical displacement $x$ of the MMSI piston is given by

$$
\frac{\pi \cdot D^{2} \cdot x}{4}=-\left(V-V_{0}\right) .
$$

Combining equation (5) with equations (3) and (4) derives the force-displacement relationship of the bearing segment as

$$
F=\frac{\pi^{2} \cdot D^{4}}{16 \cdot \beta \cdot V_{0}} x
$$

Define the segment $P_{c 1} \leq P \leq P_{c 2}$ as the working segment. There the hydraulic pressure can overcome the precompressed force, and each accumulator piston will produce an extra displacement $\Delta l(0 \leq \Delta l \leq \delta)$.

$$
\Delta l=\frac{\left[\left(P-P_{0}\right) \cdot \pi \cdot d^{2} / 4-F_{\mathrm{p}}\right]}{k} .
$$

The displacement of the MMSI piston is produced by both the volume change of the liquid and the displacement of accumulator pistons. Then the vertical displacement $x$ of the MMSI piston is given by

$$
\frac{\pi \cdot D^{2} \cdot x}{4}=-\frac{\left(V-V_{0}\right)+n \cdot \pi \cdot d^{2} \cdot \Delta l}{4} .
$$

Combining equations (7) and (8) with equations (3) and (4) derives the force-displacement relationship of the working segment as

$$
F=\frac{k \cdot x+n \cdot F_{\mathrm{P}} \cdot d^{2} / D^{2}}{16 \cdot \beta \cdot V_{0} \cdot k /\left(\pi^{2} \cdot D^{4}\right)+n \cdot d^{4} / D^{4}} .
$$

Define the segment $P_{c 2}<P$ as the limit segment. There the hydraulic pressure already pushes all accumulator pistons reaching the maximum displacement $\delta$. The displacement of the MMSI piston is produced by both the volume change of the liquid and the maximum space all accumulator pistons can offer. Then the vertical displacement $x$ of the MMSI piston is given by 


$$
\frac{\pi \cdot D^{2} \cdot x}{4}=-\frac{\left(V-V_{0}\right)+\pi \cdot d^{2} \cdot \delta}{4} .
$$

Combining equation (10) with equations (3) and (4) derives the force-displacement relationship of the limit segment as

$$
F=\frac{\pi^{2} \cdot D^{4} \cdot x-n \cdot \pi^{2} \cdot D^{2} \cdot d^{2} \cdot \delta}{16 \cdot \beta \cdot V_{0}} .
$$

Thus, the force-displacement relationship of the MMSI can be written as

$$
F= \begin{cases}\frac{\pi^{2} \cdot D^{4}}{16 \cdot \beta \cdot V_{0}} x, & P<P_{c 1}, \\ \frac{k \cdot x+n \cdot F_{P} \cdot d^{2} / D^{2}}{16 \cdot \beta \cdot V_{0} \cdot k /\left(\pi^{2} \cdot D^{4}\right)+n \cdot d^{4} / D^{4}}, & P_{c 1} \leq P \leq P_{c 2}, \\ \frac{\pi^{2} \cdot D^{4} \cdot x-n \cdot \pi^{2} \cdot D^{2} \cdot d^{2} \cdot \delta}{16 \cdot \beta \cdot V_{0}}, & P_{c 1}<P .\end{cases}
$$

Define the bearing stiffness or the limit stiffness as

$$
k_{0}=\frac{\pi^{2} \cdot D^{4}}{16 \cdot \beta \cdot V_{0}} .
$$

Define a geometrical parameter piston diameter ratio as

$$
\mu=\frac{D}{d} \text {. }
$$

Define an equivalent stiffness as

$$
k_{\mathrm{e}}=\frac{\mu^{4} \cdot k}{n} \text {. }
$$

Define the working stiffness as

$$
k_{\mathrm{w}}=\frac{k_{\mathrm{e}} \cdot k_{0}}{k_{\mathrm{e}}+k_{0}} .
$$

Define the length of the working segment as

$$
l=\left(1+\frac{k_{\mathrm{e}}}{k_{0}}\right) \cdot \frac{n \cdot \delta}{\mu^{2}} .
$$

Therefore, the force-displacement relationship of the MMSI can be rewritten as

$$
F= \begin{cases}k_{0} \cdot x, & x \leq \frac{\mu^{2} \cdot F_{\mathrm{p}}}{k_{0}}, \\ k_{\mathrm{w}} \cdot x+\frac{k_{\mathrm{w}}}{k_{\mathrm{e}}} \cdot \mu^{2} \cdot F_{\mathrm{p}}, & \frac{\mu^{2} \cdot F_{\mathrm{p}}}{k_{0}}<x \leq \frac{\mu^{2} \cdot F_{\mathrm{p}}}{k_{0}}+l, \\ k_{0} \cdot x-k_{0} \cdot \frac{n \cdot \delta}{\mu^{2}}, & \frac{\mu^{2} \cdot F_{\mathrm{p}}}{k_{0}}+l<x .\end{cases}
$$

The MMSI presents a piecewise linear force-displacement relationship according to equation (18). The bearing stiffness is equal to the limit stiffness. The bearing stiffness or the limit stiffness refers to the stiffness of the liquid spring, which is formed by only the piston-cylinder container filled with the liquid and without any accumulator. An ordinarily high bearing stiffness or the limit stiffness is because of the low compressibility of the liquid, which ensures a relatively high load capacity. As a result, the payload only needs a small static displacement to reach the working segment. The working stiffness can be regarded as the series connection of an equivalent spring with the liquid spring according to equation (16). In particular, the equivalent spring stiffness is only in connection with the configuration of accumulators according to equation (15). Moreover, concerning (17), the length of the working segment is also quite related to the configuration of accumulators. The configuration of accumulators plays a quite critical fundamental function on the MMSI HSLDS property.

2.2. MMSI Dynamical Model. It supposes that the payload locates exactly at the center of the working segment, and a linear viscous damper is added in parallel with the MMSI. Without loss of generality, the cosine wave is applied to express the harmonic excitation, so that the equation of motion of the MMSI vibration isolation system under harmonic excitation is given by

$$
M \cdot \ddot{x}+C \cdot \dot{x}+F_{k}(x)=F_{0} \cdot \cos \omega \mathrm{t},
$$

where

$$
F_{k}(x)= \begin{cases}k_{0} \cdot x+\left(k_{0}-k_{\mathrm{w}}\right) \cdot \frac{l}{2}, & x \leq-\frac{l}{2}, \\ k_{\mathrm{w}} \cdot x, & -\frac{l}{2}<x \leq \frac{l}{2}, \\ k_{0} \cdot x-\left(k_{0}-k_{\mathrm{w}}\right) \cdot \frac{l}{2}, & \frac{l}{2}<x .\end{cases}
$$

It is possible to obtain an exact quantitative solution by utilizing piecewise analytic integral as for a piecewise linear system. Meanwhile applying the averaging method is a simpler and convenient way to achieve the quantitative solution when the accuracy requirement is limited to the first-order term of the nonlinear factor [17].

Define the nonlinear factor as $\varepsilon=\left(k_{0} / k_{\mathrm{w}}\right)-1$; and let $\omega_{0}^{2}=k_{\mathrm{w}} / M, \quad \xi=\varepsilon \cdot \xi_{1}=\left(C /\left(2 \cdot \sqrt{M \cdot k_{\mathrm{w}}}\right)\right), \quad f_{0}=\varepsilon \cdot f_{1}=$ $F_{0} / M$, and $\omega^{2}=\omega_{0}^{2} \cdot(1+\varepsilon \cdot \sigma)$, where $\sigma$ is a detuning parameter. Define a piecewise function as follows:

$$
f_{k}(x)=\left\{\begin{array}{cc}
x+\frac{l}{2}, & x \leq-\frac{l}{2}, \\
0, & -\frac{l}{2}<x \leq \frac{l}{2}, \\
x-\frac{l}{2}, & \frac{l}{2}<x .
\end{array}\right.
$$


Then the equation of motion can be rewritten in the dimensionless form as

$$
\ddot{x}+\omega^{2} \cdot x=\varepsilon \cdot f(x, \dot{x}, \omega t),
$$

where

$$
f(x, \dot{x}, \omega t)=f_{1} \cdot \cos \omega t-2 \cdot \xi_{1} \cdot \omega_{0} \cdot \dot{x}+\omega_{0}^{2} \cdot\left(\sigma \cdot x-f_{k}(x)\right) .
$$

In particular, when $\varepsilon=0$, the solution is given by

$$
\begin{aligned}
& x=a \cdot \cos (\omega t-\theta), \\
& \dot{x}=-a \cdot \omega \cdot \sin (\omega t-\theta),
\end{aligned}
$$

where $a$ and $\theta$ are constants. However, when $\varepsilon \neq 0$, the solution can still be represented by that solution, but $a$ and $\theta$ are functions with respect to $t$ rather than constants. By introducing $\psi=\omega t-\theta$ and substituting $\psi$ into the solution and then differentiating the solution with respect to $t$, we obtain

$$
\begin{aligned}
& \dot{x}=\dot{a} \cdot \cos \psi-a \cdot(\omega-\dot{\theta}) \cdot \sin \psi, \\
& \ddot{x}=-\dot{a} \cdot \omega \cdot \sin \psi-a \cdot \omega \cdot(\omega-\dot{\theta}) \cdot \cos \psi .
\end{aligned}
$$
derives

Combining equations (24) and (25) with equation (22)

$$
\begin{aligned}
\dot{a} \cdot \cos \psi+a \cdot \dot{\theta} \cdot \sin \psi & =0 \\
-\dot{a} \cdot \sin \psi+a \cdot \dot{\theta} \cdot \cos \psi & =\frac{\varepsilon}{\omega} \cdot f(x, \dot{x}, \omega t) .
\end{aligned}
$$

According to the equation set of (26), we can obtain two differential equations of $a$ and $\theta$ as

$$
\begin{aligned}
& \dot{a}=-\frac{\varepsilon}{\omega} \cdot f(x, \dot{x}, \omega t) \cdot \sin \psi, \\
& \dot{\theta}=\frac{\varepsilon}{\omega \cdot a} \cdot f(x, \dot{x}, \omega t) \cdot \cos \psi .
\end{aligned}
$$

Equation (27) denotes that $\dot{a}$ and $\dot{\theta}$ have the same order as the parameter $\varepsilon$, and they are slowly varying functions compared to the function $\dot{\psi}$. It is assumed that the right term of equation (27) can be approximately alternated by its average over a period of $\psi$, and $a$ and $\theta$ are constants in that period. As a result, the averaging equation can be achieved as

$$
\begin{aligned}
\dot{a} & =-\frac{\varepsilon}{2 \cdot \pi \cdot \omega} \cdot \int_{0}^{2 \pi} f(x, \dot{x}, \omega t) \cdot \sin \psi \mathrm{d} \psi \\
& =-\frac{\varepsilon}{2 \cdot \omega} \cdot\left(2 \cdot a \cdot \xi_{1} \cdot \omega_{0} \cdot \omega-f_{1} \cdot \sin \theta\right) \\
\dot{\theta} & =\frac{\varepsilon}{2 \cdot \pi \cdot \omega \cdot a} \cdot \int_{0}^{2 \pi} f(x, \dot{x}, \omega t) \cdot \cos \psi \mathrm{d} \psi \\
& =\frac{\varepsilon}{2 \cdot \omega \cdot a} \cdot\left(-\frac{2 \cdot a \cdot \omega_{0}^{2}}{\pi} \cdot G(\alpha)+\omega_{0}^{2} \cdot \sigma \cdot a+f_{1} \cdot \cos \theta\right),
\end{aligned}
$$

where $\alpha=(2 \cdot a) / l$ and

$$
G(\alpha)= \begin{cases}1, & \alpha \leq 1, \\ \arccos \frac{1}{\alpha}-\frac{1}{\alpha} \cdot \sqrt{1-\left(\frac{1}{\alpha}\right)^{2}}, & 1<\alpha .\end{cases}
$$

In order to achieve the steady primary resonance response, letting $\dot{a}=0$ and $\dot{\theta}=0$, we can obtain the amplitudefrequency equation and phase-frequency equation as

$$
\begin{aligned}
& \alpha^{2} \cdot\left\{\left[1-s^{2}+\frac{2 \cdot \varepsilon}{\pi} \cdot G(\alpha)\right]^{2}+(2 \cdot \xi \cdot s)^{2}\right\}=B^{2} \\
& \theta=\arctan \frac{2 \cdot \xi \cdot s}{1-s^{2}+((2 \cdot \varepsilon) / \pi) \cdot G(\alpha)},
\end{aligned}
$$

where $B=\left(2 \cdot f_{0}\right) /\left(\omega_{0}^{2} \cdot l\right)$ and $s=\omega / \omega_{0}$.

2.3. Energy Transmissibility (ET). In the conventional linear vibration isolation theory, force transmissibility is often applied to the vibration isolation performance index. Concerning a nonlinear system, the nonlinear steady response may have some nonexcitation frequency including subharmonics or superharmonics. The force transmissibility may not be applicable to that index. Some literatures $[13,14,18]$ mentioned that the energy transmissibility may be suitable for the vibration isolation performance index. The energy transmissibility is introduced and defined as

$$
\eta=20 \cdot \lg \sqrt{\frac{P_{1}}{P_{0}}},
$$

where $P_{1}$ and $P_{0}$ are power of transmission force and excitation force, respectively, which can be evaluated by

$$
\begin{aligned}
P_{1} & =\frac{\omega}{2 \cdot \pi} \cdot \int_{0}^{(2 \cdot \pi) / \omega}\left(C \cdot \dot{x}+F_{k}(x)\right)^{2} \mathrm{~d} t \\
& =\frac{\omega}{2 \cdot \pi} \cdot \int_{0}^{(2 \cdot \pi) / \omega}\left(F_{0} \cdot \cos \omega t-M \cdot \ddot{x}\right)^{2} \mathrm{~d} t, \\
P_{0} & =\frac{\omega}{2 \cdot \pi} \cdot \int_{0}^{(2 \cdot \pi) / \omega}\left(F_{0} \cdot \cos \omega t\right)^{2} \mathrm{~d} t .
\end{aligned}
$$

Equation (32) shows that the power of the transmission force can be evaluated in two ways. The first approach needs the displacement and the velocity, which can be obtained with the averaging method theoretically. Meanwhile, as for an experimental test, the second approach is more convenient for the acceleration is easier to be measured.

The ET essentially signifies the ratio of root-mean-square of transmitted force over that of external excitation force. It is a generalised form of the force transmissibility. Similarly, if the ET is less than zero, the isolator has positive performance of reducing the energy transmitted from the excitation source to the base.

\section{Experimental Apparatus and Results}

An experimental apparatus was designed to validate the MMSI stiffness model; moreover, dynamic experiments were carried out to estimate its vibration isolation 


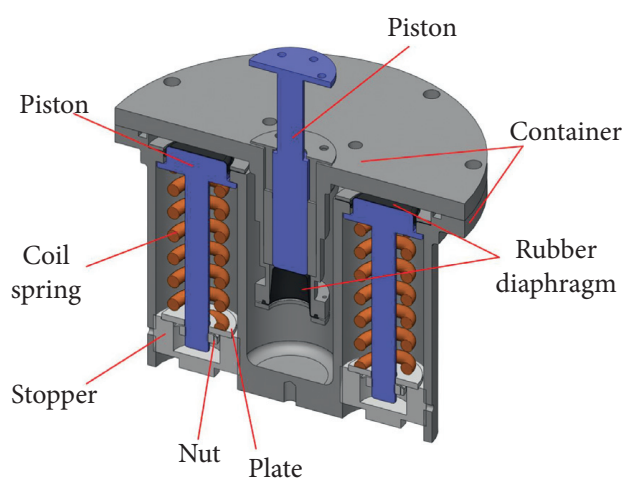

(a)

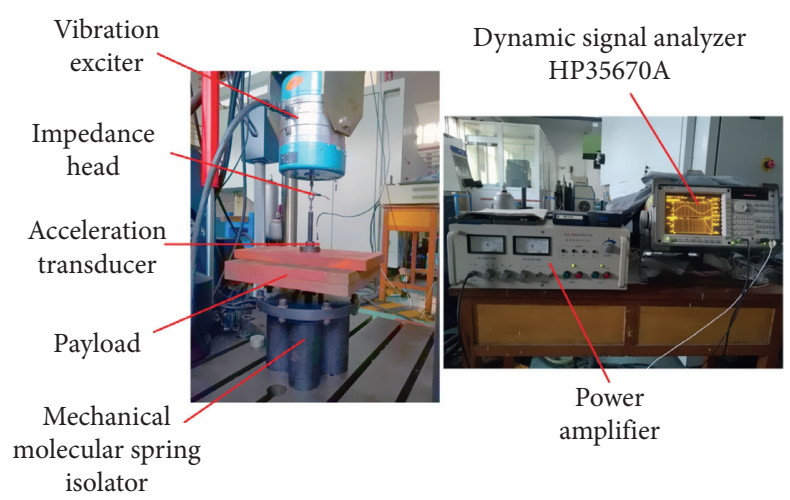

(b)

Figure 2: Experimental apparatus. (a) Section view of the MMSI. (b) Dynamic experiment setup.

performance. The section view of the experimental apparatus 3D model is plotted in Figure 2(a). The apparatus consists of four identical accumulators that are embedded within the piston-cylinder container rather than being independent. The container is filled with water so as to intercommunicate all accumulators. The pressure on water may pass to the piston of all accumulators instantly and simultaneously. Each accumulator includes a stair-step piston, a coil spring, a plate, a nut, and a stopper. The piston is confined by the container, the coil spring, and the stopper. It is noticed that the container piston and accumulator pistons are sealed by rubber diaphragms, which may not introduce any friction compared to the conventional seal ring. The container piston is oriented by a ball guide sleeve, while each accumulator piston is oriented by the precompressed force and the rubber diaphragm automatically.

The static stiffness property of the MMSI was tested with quasi-static experiments on a fatigue testing machine. The dynamic experiments were carried out with a vibration exciter. The dynamic experimental setup is presented in Figure 2(b); the vibration exciter was fastened on the upper clamping head of the fatigue testing machine, and the MMSI was mounted on the ground. Meanwhile, the payload was fixed on the piston by a threaded rod; the impedance head was fixed between the vibration exciter and the threaded rod by two connecting rods; the acceleration transducer was pasted on the payload. The vibration exciter was controlled by a power amplifier, which was connecting with the dynamic signal analyzer HP35670A. The force and acceleration signals gathered by the impedance head and the acceleration transducer were delivered to the dynamic signal analyzer HP35670A. It employed the sweep sine mode to proceeding dynamic experiments.

Basic parameters of the experimental apparatus applied to the theoretical analysis are listed in Table 1 . It is noted that the definition of the effective diameter of a piston is extracted from the theory of rubber diaphragm [19]. As a result, according to the theory above, the theoretical stiffnesses of the bearing segment and the working segment can be derived, and they are listed in the last column of Table 2. The theoretical force-displacement figures are plotted in Figure 3(a). Those curves marked " 0 ," "1," "2," " 3 ," and "4"
TABle 1: Parameters of the experimental apparatus.

\begin{tabular}{lc}
\hline Parameters & Value \\
\hline $\begin{array}{l}\text { The effective diameter of the } \\
\text { container piston } D\end{array}$ & $32.5 \mathrm{~mm}$ \\
The effective diameter of the & $54.5 \mathrm{~mm}$ \\
accumulator piston $d$ & $825 \mathrm{~mL}$ \\
The initial volume of the water $V_{0}$ & $1.9 e 5 \mathrm{~N} / \mathrm{m}$ \\
The average stiffness of coil springs $k$ & $1466.5 \mathrm{~N}$ \\
The average precompressed & $2.00 \mathrm{~mm}$ \\
force within coil springs $F_{\mathrm{p}}$ & 4 \\
The average maximum displacement $\delta$ & $60 \mathrm{~kg}$ \\
The number of hydraulic & \\
spring accumulators $n$ &
\end{tabular}

mean that there are none, one, two, three, and four accumulators being engaged in the isolator. In particular, the curve marked "0" acts as the traditional linear counterpart of those MMSIs with different accumulators. On account of the precompressed force on all identical accumulators, Figure 3(a) shows that the MMSI presents a piecewise linear property of three segments. All curves of MMSIs have the same turning point, which denotes the transformation from the bearing segment to the working segment. However, concerning the transformation from the working segment to the limit segment, they turn at the same force but not at the same displacement. What is more, apparently, the working segment's stiffness gets lower significantly as the number of accumulators increases; however, the working segment's length is widened. In the initial design, the payload is expected to be balanced at the center of the working segment of all MMSIs.

A series of quasi-static experiments on isolators with different number of accumulators were conducted. The loading process and the unloading process are presented in Figure 3(b). Compared to theoretical curves on Figure 3(a), the most obvious difference lies in two points. First, the experimental results show a distinct noncoincidence of the loading process and the unloading process. Second, the experimental slope of the bearing segment is much less than the theoretical one. Looking back to the experimental apparatus as shown in Figure 2(a), we found that only rubber diaphragms may produce both effects on 
TABLE 2: The working stiffness of the MMSI.

\begin{tabular}{lcccc}
\hline Number of accumulators & Loading stiffness $(\mathrm{N} / \mathrm{m})$ & Unloading stiffness $(\mathrm{N} / \mathrm{m})$ & Average stiffness $(\mathrm{N} / \mathrm{m})$ & Theoretical stiffness $(\mathrm{N} / \mathrm{m})$ \\
\hline 0 & $2.83 e 5$ & $3.57 e 5$ & $3.20 e 5$ & $1.84 e 6$ \\
1 & $2.21 e 4$ & $2.43 e 4$ & $2.32 e 4$ & $2.20 e 4$ \\
2 & $1.29 e 4$ & $1.26 e 4$ & $1.28 e 4$ & $1.14 e 4$ \\
3 & $9.04 e 3$ & $8.20 e 3$ & $8.52 e 3$ & $7.69 e 3$ \\
4 & $6.87 e 3$ & $6.21 e 3$ & $6.54 e 3$ & $5.80 e 3$ \\
\hline
\end{tabular}

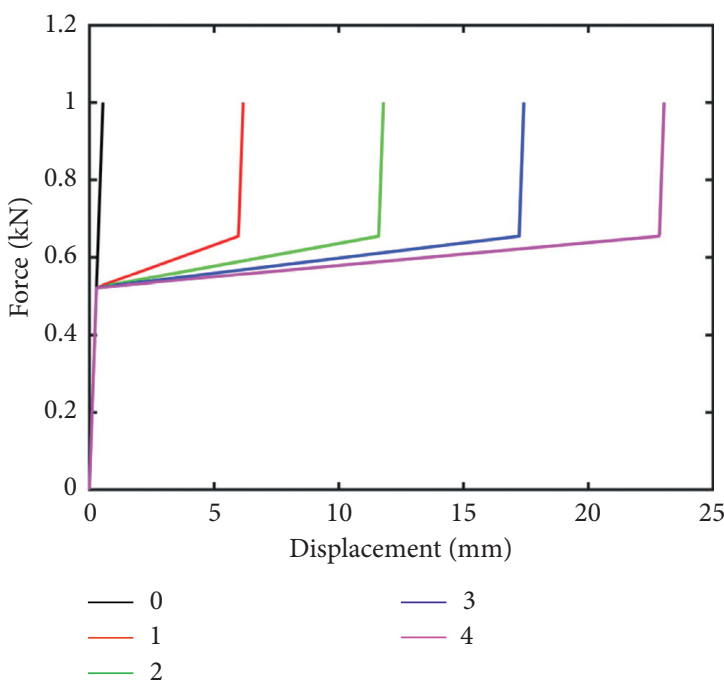

(a)

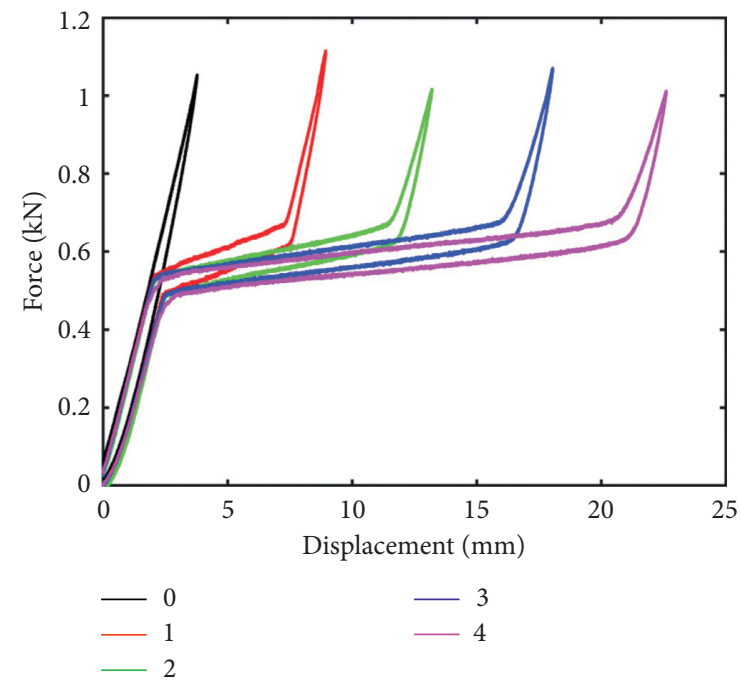

(b)

FIgURE 3: Stiffness property of the MMSI. (a) Theoretical force-displacement relationships of the MMSI with different number of elements. (b) Experimental force-displacement relationships of the MMSI with different number of elements.

the MMSI stiffness property. First, the rubber diaphragm is inevitable to get some elastic deformation to satisfy the inner water pressure. Second, the rubber in the rubber diaphragm may get some plastic deformation when the loading is much higher so as to produce some hysteresis [19]. Even so, the experimental working stiffnesses of MMSIs fit well with the theoretical one according to Table 2, in which the loading working stiffnesses and the unloading working stiffnesses were extracted from Figure 3(a) and the average stiffnesses of both are listed.

After quasi-static experiments, the dynamic experiments were in procedure. In the first place, the theoretical ETs of the isolation system with different isolators were worked out. Based on the experimental apparatus, it neglects the damping in each accumulator while only considering the damping on the piston that supports the payload to conduct the theory calculation. It supposes that there is a linear damping on the piston with the damping coefficient of $200 \mathrm{Ns} / \mathrm{m}$. As shown in Figure 4(a), when the isolation system is under the same relatively low excitation force of $10 \mathrm{~N}$, all dynamic responses appear like a linear system. Meanwhile, compared to the conventional counterpart, the system applying the MMSI with different number of accumulators shows a distinct benefit in the initial frequency of the isolation bandwidth. On the contrary, when the excitation force reaches a relatively higher value of $20 \mathrm{~N}$, an inevitable jump phenomenon may occur according to Figure 4(b). This is a quite common phenomenon for an HSLDS vibration isolator. In the second place, the experimental ETs were also obtained correspondingly. In the test process, we adjusted the magnitude of the power amplifier to change the excitation force. A relatively low signal of $0.5 \mathrm{~V}$ produced from power amplifier was applied to conduct the dynamic experiments at first; and the results are shown in Figure 4(c). In comparison with the theoretical results of Figure 4(a), firstly, they both demonstrated that the MMSI is superior to the conventional counterpart in isolating a lower frequency vibration. Secondly, the MMSI possessing more accumulators is better for isolating a much lower frequency vibration. Thirdly, the curves marked " 0 ," “ 1 ," " 2 ," “ 3 ," or " 4 " dramatically do not fit well. This is because of the application of rubber diaphragm, which may produce much more elastic deformation than the liquid and some plastic deformation. A relatively high signal of $1.0 \mathrm{~V}$ was applied to the test to verify the jump phenomenon. As shown in Figure 4(d), the results of both sequential and backward sweeps on the system with the MMSI of one accumulator are presented. It is clear that both results share the low- and high-frequency response, while they do not have the same jump frequency around the resonance frequency. These are the typical experimental results of a system with jump phenomenon. 


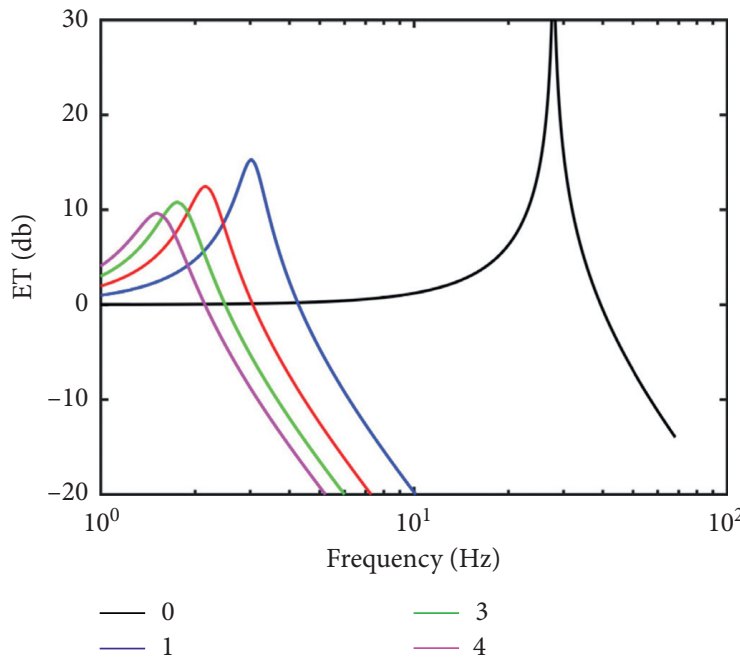

(a)

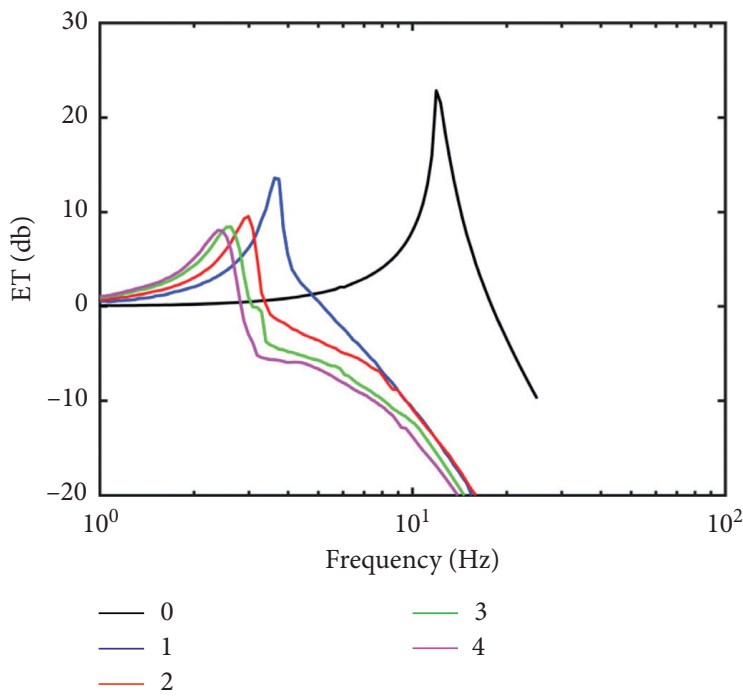

(c)

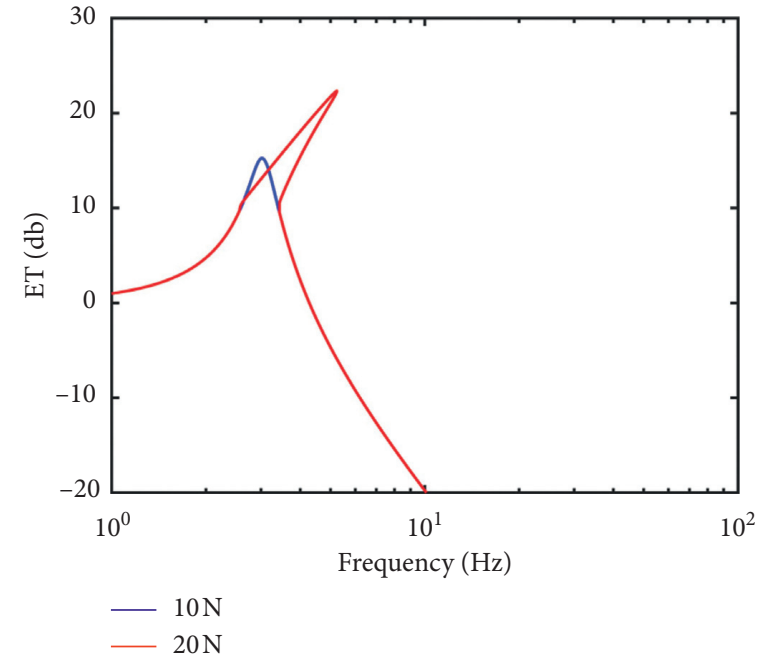

(b)

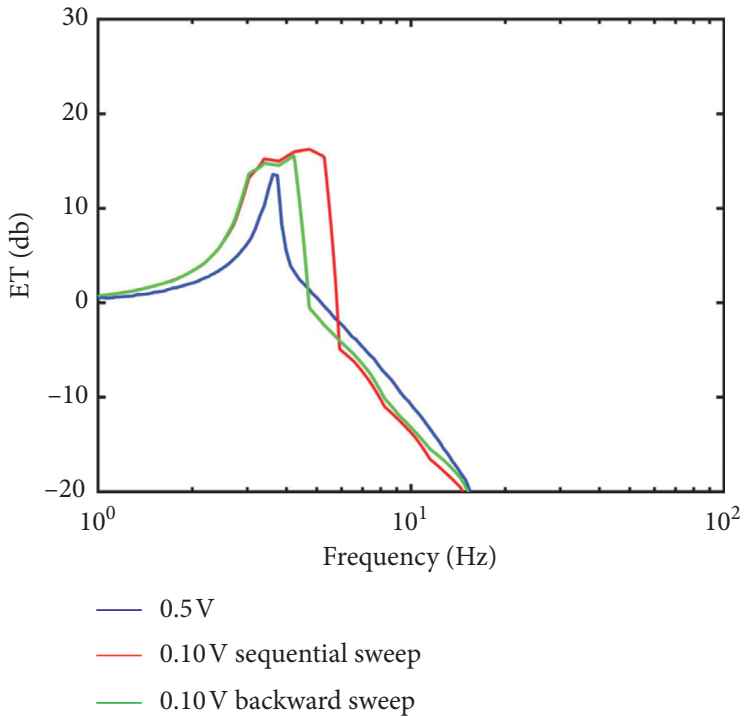

(d)

FIGURE 4: ETs of the MMSI vibration isolation system. (a) Theoretical ETs of the system with different number of accumulators under the same excitation force $(10 \mathrm{~N})$. (b) Theoretical ETs of the system with one accumulator under different excitation forces $(10 \mathrm{~N}$ and $35 \mathrm{~N})$. (c) Experimental ETs of the system with different number of accumulators under the same excitation force (0.5 V). (d) Experimental ETs of the system with one accumulator under different excitation forces $(0.5 \mathrm{~V}$ and $1 \mathrm{~V})$.

However, there are also some weird properties in contrast to the theoretical results in Figure 4(d), where the experimental results of a higher excitation force get shifted to the left compared to the lower one. We also attribute the mismatching results to the application of the rubber diaphragm. After all, the MMSI is proved to be a typical HSLDS vibration isolator.

\section{Conclusions}

This article focused on the stiffness model of the MMSI with several identical accumulators. It found that the stiffness model of MMSI presents a piecewise linear HSLDS property, while the working stiffness was mainly determined by the structure of the accumulator. The working stiffness is biquadratic to the diameter ratio of pistons, directly proportional to the linear spring stiffness, and inversely proportional to the number of accumulators. The length of the working segment is directly proportional to the number of accumulators and the maximum displacement and inversely proportional to the square of the diameter ratio of pistons. A proper design of the length of the segment may allow the payload to always vibrate within the working segment without introducing any nonlinear dynamic phenomenon. Besides, it applies the averaging method to achieve the approximate frequency responses and utilizes the energy transmissibility to evaluate the MMSI vibration isolation system performance. 
An MMSI prototype has been designed to carry out the static and dynamic experiments. The experimental static working stiffnesses basically follow the theoretical principles. Dynamic experimental results indicate that MMSI outperforms the linear counterpart, and it also shows a wider vibration isolation bandwidth with the increase of the number of accumulators. The typical nonlinear dynamic phenomenon sudden jump, which is caused by the inherent characteristic of an isolator with HSLDS, happened when the excitation force came to the critical level.

However, there are still several aspects of this design that require further investigation. For example, the configurations of all accumulators can be designed with different types, though it is still for this kind, three-segmented HSLDS property, or some other kinds. Moreover, it is able to take some advantages of the fluid to introduce the hydraulic system to take an active control on the isolation system.

\section{Data Availability}

The experimental data used to support the findings of this study are available from the corresponding author upon request.

\section{Conflicts of Interest}

The authors declare that there are no conflicts of interest regarding the publication of this paper.

\section{Acknowledgments}

The authors would like to thank Dr. Shuai Zhao for his assistance with the experimental design and thank Dr. Fangshuo Li for his helpful advice. This work was supported by the National Natural Science Foundation of China under Grant no. 11472127.

\section{References}

[1] S. S. Rao, Mechanical Vibrations, Prentice Hall, Upper Saddle River, NJ, USA, 5th edition, 2011.

[2] E. I. Rivin, Passive Vibration Isolation, ASME Press, New York, NY, USA, 2003.

[3] C. M. Harris and A. G. Piersol, Harris' Shock and Vibration Handbook, McGraw-Hill, New York, NY, USA, 5th edition, 2002.

[4] A. Carrella, "Passive vibration isolators with high-static-lowdynamic-stiffness (HSLDS)," Ph.D. thesis, University of Southampton, Southampton, UK, 2008.

[5] I. Kovacic, M. J. Brennan, and T. P. Waters, "A study of a nonlinear vibration isolator with a quasi-zero stiffness characteristic," Journal of Sound and Vibration, vol. 315, no. 3, pp. 700-711, 2008.

[6] A. Carrella, M. J. Brennan, and T. P. Waters, "Static analysis of a passive vibration isolator with quasi-zero-stiffness characteristic," Journal of Sound and Vibration, vol. 301, no. 3-5, pp. 678-689, 2007.

[7] A. Carrella, M. J. Brennan, I. Kovacic, and T. P. Waters, "On the force transmissibility of a vibration isolator with quasizero-stiffness," Journal of Sound and Vibration, vol. 322, no. 45, pp. 707-717, 2009.
[8] A. Carrella, M. J. Brennan, T. P. Waters, and K. Shin, "On the design of a high-static-low-dynamic stiffness isolator using linear mechanical springs and magnets," Journal of Sound and Vibration, vol. 315, no. 3, pp. 712-720, 2008.

[9] X. Huang, X. Liu, J. Sun, Z. Zhang, and H. Hua, "Vibration isolation characteristics of a nonlinear isolator using Euler buckled beam as negative stiffness corrector: a theoretical and experimental study," Journal of Sound and Vibration, vol. 333, no. 4, pp. 1132-1148, 2014.

[10] J. Zhou, X. Wang, D. Xu, and S. Bishop, "Nonlinear dynamic characteristics of a quasi-zero stiffness vibration isolator with cam-roller-spring mechanisms," Journal of Sound and Vibration, vol. 346, pp. 53-69, 2015.

[11] X. Sun and X. Jing, "Multi-direction vibration isolation with quasi-zero stiffness by employing geometrical nonlinearity," Mechanical Systems and Signal Processing, vol. 62-63, pp. 149-163, 2015.

[12] S. M. M. Mofidian and H. Bardaweel, "Displacement transmissibility evaluation of vibration isolation system employing nonlinear-damping and nonlinear-stiffness elements," Journal of Vibration and Control, vol. 24, no. 18, pp. 4247-4259, 2018.

[13] H. D. Teng and Q. Chen, "Study on vibration isolation properties of solid and liquid mixture," Journal of Sound and Vibration, vol. 326, no. 1-2, pp. 137-149, 2009.

[14] X. Gao, Q. Chen, and H. D. Teng, "Modelling and dynamic properties of a novel solid and liquid mixture vibration isolator," Journal of Sound and Vibration, vol. 331, no. 16, pp. 3695-3709, 2012.

[15] M. Yu, X. Gao, and Q. Chen, "Study of the mechanical properties and vibration isolation performance of a molecular spring isolator," Shock and Vibration, vol. 2016, Article ID 6451829, 10 pages, 2016.

[16] M. C. Yu, Q. Chen, and X. Gao, "Theoretical and experimental investigation of molecular spring isolator," Applied Mechanics and Materials, vol. 764-765, pp. 388-392, 2015.

[17] Y. Z. Liu and L. Q. Chen, Nonlinear Vibrations, Higher Education Press, Beijing, China, 2001.

[18] J.-j. Lou, S.-j. Zhu, L. He, and X. Yu, "Application of chaos method to line spectra reduction," Journal of Sound and Vibration, vol. 286, no. 3, pp. 645-652, 2005.

[19] Diacom, Diaphragm Design Guidebook, Diacom Corporation, Amherst, NH, USA, 2008, http://www.diacom.com. 